- Original Article

\title{
Correlation of Arterial Stiffness and Bone Mineral Density by Measuring Brachial-Ankle Pulse Wave Velocity in Healthy Korean Women
}

\author{
Nam-Lee Kim, Heuy-Sun Suh* \\ Department of Family Medicine, Gachon University Gil Medical Center, Incheon, Korea
}

Background: An association between arterial stiffness and osteoporosis has previously been reported. Therefore, we investigated the relationship between arterial stiffness, measured by brachial-ankle pulse wave velocity, and bone mineral density in a sample of healthy women undergoing routine medical checkup.

Methods: We retrospectively reviewed the medical charts of 135 women who had visited the Health Promotion Center (between May 2009 and December 2012). Brachial-ankle pulse wave velocity was measured using an automatic wave analyzer. Bone mineral density of the lumbar spine (L1-L4) and femur was measured by dual-energy Xray absorptiometry. Metabolic syndrome was defined according to National Cholesterol Education Program-Adult Treatment Panel III criteria, using body mass index $>25 \mathrm{~kg} / \mathrm{m}^{2}$ instead of waist circumference $>88.9 \mathrm{~cm}$.

Results: Pearson's correlation analysis revealed significant inverse relationships between pulse wave velocity and bone mineral density of the lumbar spine $(\mathrm{r}=-0.335, \mathrm{P}<0.001)$, femur neck $(\mathrm{r}=-0.335, \mathrm{P}<0.001)$, and total femur $(\mathrm{r}=-0.181, \mathrm{P}=0.04)$. Pulse wave velocity showed the strongest association with age $(\mathrm{r}=0.586, \mathrm{P}<0.001)$. Multiple regression analysis identified an independent relationship between pulse wave velocity and lumbar spine bone mineral density in women after adjusting for age, metabolic syndrome, body mass index, smoking status, alcohol intake, and exercise $(\mathrm{r}=-0.229, \mathrm{P}=0.01)$.

Conclusion: This study confirmed an association between arterial stiffness and bone mineral density in women.

Keywords: Bone Density; Osteoporosis; Pulse Wave Analysis; Vascular Stiffness 


\section{INTRODUCTION}

Cardiovascular disease (CVD) and osteoporosis are two of the major causes of death and illness in the elderly. Some researchers have found that these conditions, the risk of both of which increases with age, are independently correlated with age. In particular, a correlation between bone mineral density (BMD) and death from CVD has been observed among postmenopausal women. ${ }^{1,2)}$ Lower BMD has been associated with a higher risk of coronary artery disease ${ }^{3,4)}$ and several studies report bone loss accompanied by vascular calcification. ${ }^{5-7)}$

Researchers have also investigated the correlation between arterial stiffness and osteoporosis. ${ }^{8-10)}$ Although there are several measures of arterial stiffness, including central blood pressure (BP) and the augmentation index (AI), pulse wave velocity (PWV) is widely used as it is convenient, noninvasive, and inexpensive. The measurement of arterial stiffness may help predict the risk of cardiovascular diseases and determine the efficacy of treatment for these conditions.

In clinical practice, brachial-ankle pulse wave velocity (baPWV), which records the pulse wave using an oscillometry sensor on brachial-ankle BP cuffs, is frequently used because it does not require sophisticated technology or exposure of the inguinal area. Recent reports indicate that ba-PWV is correlated with carotid-femoral PWV; ${ }^{11,12)}$ although the former of the two methods has been less extensively researched, it was found to be useful in predicting CVD mortality among patients with cardiovascular diseases, renal failure, or heart failure. ${ }^{13-15)}$ Vascular calcification can cause arterial stiffness, and both PWV and vascular calcification may reflect atherosclerosis.

The correlation between osteoporosis and CVD or CVD risk factors, such as atherosclerosis, arterial stiffness, and vascular calcification, is still controversial and findings concerning this correlation are not consistent with one another. Here, we aimed to use ba-PWV to analyze the relationship between arterial stiffness and BMD of both, the lumbar spine (L-spine) and hip (femur) joints in healthy women undergoing routine medical checkup.

\section{METHODS}

\section{Subjects}

Medical charts were analyzed retrospectively for 135 women who had undergone routine medical checkup at the Health Promotion Center (between May 2009 and December 2012). We reviewed the patient-doctor consultation records for information about each patient's lifestyle habits, medical history, previous medication, smoking status, and alcohol intake. Patients were asked to take off their outer garment before their height and weight were measured on a single height and weight scale; body mass index (BMI) was calculated on the basis of weight (kg) and height (m). For all patients, the basic regular checkup comprised a blood test, ba-PWV, and dual-energy Xray absorptiometry (L-spine and femur). The blood test was performed after a $\geq 12$-hour fast and BP was measured using an electronic manometer on the right arm after a $\geq 10$-minute rest. Women who had a medical history of hypertension, diabetes, hyperlipidemia, chronic renal failure, thyroid disease, parathyroid disease, or rheumatoid arthritis, who had used bisphosphonate or steroid medications, or who were diagnosed with cancer less than five years prior were excluded from the study.

\section{Clinical Measurements}

BMD was measured in the L-spine (L1-L4), femur neck, and total femur using dual-energy X-ray absorptiometry (Lunar Prodigy Advance; GE Healthcare Technologies, Madison, WI, USA). The results were presented in $\mathrm{g} / \mathrm{cm}^{2}$.

Patients were asked to rest in the supine position for $10 \mathrm{~min}$ utes before PWV was measured using an automatic waveform analyzer (VP-1000; Clin Co., Komaki, Japan). Oscillometry was used to measure BP in both arms (posterior tibial artery) and ankles (brachial artery); the ankle-brachial index was calculated automatically. Right and left ba-PWVs were measured simultaneously, and the mean of both ba-PWVs was used for all analyses.

Patients were categorized into groups on the basis of their CVD risk factors: smoking group (current smokers) versus nonsmoking group (including former smokers); drinking group (patients consuming $\geq 1.5$ bottles of alcohol per week) versus nondrinking group (patients drinking $<1.5$ bottles of alcohol per week); exercise group (patients exercising for $\geq 30$ minutes per day or $\geq 3$ times a week) versus no-exercise group (patients exercising for $<30$ minutes per day or $\leq 3$ times a week).

We defined metabolic syndrome in accordance with the guidelines of the 2000 World Health Organization Recommendation for Obesity Standards in Asian-Pacific Regions and the National Cholesterol Education Program-Adult Treatment Panel III, ${ }^{16)}$ referring to the patient blood test results and replacing the waist circumference criteria ( $>88.9 \mathrm{~cm}$ for women) with BMI $>25 \mathrm{~kg} / \mathrm{m}^{2}$. Those patients satisfying $\geq 3$ of the following diagnostic criteria were considered to have metabolic syndrome: (1) $\mathrm{BMI} \geq 25 \mathrm{~kg} / \mathrm{m}^{2}$; (2) fasting triglycerides $\geq 150 \mathrm{mg} / \mathrm{dL}$; (3) fasting high density lipoprotein cholesterol $<50 \mathrm{mg} / \mathrm{dL}$; (4) fasting blood glucose $\geq 100 \mathrm{mg} / \mathrm{dL}$; and (5) BP $\geq 130 / 85$ mm Hg.

\section{Statistical Analysis}

All variables were presented as mean \pm standard deviation. Independent $t$-tests were used to analyze variation in ba-PWV by metabolic syndrome status, alcohol intake, smoking status, and exercise habits. Pearson correlation analysis was performed to evaluate the relationship between age, BMI, and re- 
Table 1. Clinical characteristics of the women in this study $(N=135)$

\begin{tabular}{lc}
\hline Characteristic & Value \\
\hline Age $(\mathrm{y})$ & $53.2 \pm 6.3$ \\
Body mass index $\left(\mathrm{kg} / \mathrm{m}^{2}\right)$ & $23.8 \pm 2.0$ \\
Systolic blood pressure $(\mathrm{mm} \mathrm{Hg})$ & $125.4 \pm 8.4$ \\
Diastolic blood pressure $(\mathrm{mm} \mathrm{Hg})$ & $81.7 \pm 10.2$ \\
Bone mineral density $\left(\mathrm{g} / \mathrm{cm}^{2}\right)$ & \\
$\quad$ Lumbar spine & $1.82 \pm 0.38$ \\
Femur neck & $0.99 \pm 0.22$ \\
Femur trochanter & $0.94 \pm 0.35$ \\
$\quad$ Femur total & $0.99 \pm 0.24$ \\
Fasting glucose (mg/dL) & $82.7 \pm 15.1$ \\
Low density lipoprotein cholesterol (mg/dL) & $110.1 \pm 20.2$ \\
High density lipoprotein cholesterol (mg/dL) & $53.0 \pm 12.7$ \\
Triglycerides (mg/dL) & $95.9 \pm 38.5$ \\
Total cholesterol (mg/dL) & $195.5 \pm 26.7$ \\
Mean pulse wave velocity (cm/s) & $1,370.5 \pm 180.5$ \\
Smoking & $8 / 135(5.9)$ \\
Alcohol & $9 / 135(6.6)$ \\
Exercise & $48 / 135(35.5)$ \\
Metabolic syndrome & $10 / 135(7.4)$ \\
\hline
\end{tabular}

Values are presented as mean \pm standard deviation or number (\%).

gional BMD and ba-PWV.

Multiple regression analysis was performed with ba-PWV as the dependent variable; all variables that showed statistically significant relationships with ba-PWV upon independent t-test and Pearson correlation analysis were included as covariables. Variables that were not significantly related to ba-PWV $(\mathrm{P}>0.05)$ but were considered to act clinically as confounding variables (alcohol intake, smoking status, exercise, and metabolic syndrome) were also included as covariables. The significance level was set at $\mathrm{P}<0.05$ and all the statistical analyses were conducted using SPSS ver. 12.0 (SPSS Inc., Chicago, IL, USA).

\section{RESULTS}

\section{Subject Characteristics}

The 135 women in this study had an average age of 53 years and mean BMI of $23 \mathrm{~kg} / \mathrm{m}^{2}$. The mean ba-PWV was $1,370.5 \pm$ $180.5 \mathrm{~cm} / \mathrm{s}$; mean values of other clinical variables and CVD risk factors are shown in Table 1.

\section{Univariate Analysis}

Independent t-tests revealed no significant differences in baPWV among women on the basis of their alcohol intake, smoking behavior, or exercise status $(\mathrm{P}=0.14, \mathrm{P}=0.65$, and $\mathrm{P}=0.42$, respectively). In contrast, the metabolic syndrome group showed significantly higher ba-PWV values than the nonmetabolic syndrome group $(1,349.16 \pm 97.62, \mathrm{P}<0.01)$ (Table was not shown).

Pearson correlation analysis revealed a significant negative correlation between ba-PWV and L-spine BMD, femur neck BMD, and total femur BMD (as shown in Table 2). In contrast,
Table 2. Correlation between brachial-ankle pulse wave velocity and clinical parameters

\begin{tabular}{lcc}
\hline Clinical parameter & Pearson's R & P-value \\
\hline Age $(\mathrm{y})$ & 0.586 & $<0.001$ \\
Lumbar spine BMD $\left(\mathrm{g} / \mathrm{cm}^{2}\right)$ & -0.335 & $<0.001$ \\
Femur neck BMD $\left(\mathrm{g} / \mathrm{cm}^{2}\right)$ & -0.289 & 0.02 \\
Femur trochanter BMD $\left(\mathrm{g} / \mathrm{cm}^{2}\right)$ & -0.123 & 0.32 \\
Femur total BMD $\left(\mathrm{g} / \mathrm{cm}^{2}\right)$ & -0.181 & 0.04 \\
Body mass index $\left(\mathrm{kg} / \mathrm{m}^{2}\right)$ & 0.092 & 0.46 \\
\hline BMD, bone mineral density. & &
\end{tabular}

Table 3. Relationships between brachial-ankle pulse wave velocity and predictor variables by multiple regression

\begin{tabular}{lcc}
\hline Variable & $\beta$ & P-value \\
\hline Age $(\mathrm{y})$ & 0.458 & $<0.001$ \\
Lumbar spine BMD $\left(\mathrm{g} / \mathrm{cm}^{2}\right)$ & -0.229 & 0.01 \\
Femur neck BMD $\left(\mathrm{g} / \mathrm{cm}^{2}\right)$ & 0.128 & 0.26 \\
Femur total BMD $\left(\mathrm{g} / \mathrm{cm}^{2}\right)$ & -0.058 & 0.56 \\
Metabolic syndrome & 0.248 & 0.001 \\
Smoking & 0.012 & 0.88 \\
Alcohol & 0.016 & 0.83 \\
Exercise & 0.034 & 0.64
\end{tabular}

BMD, bone mineral density.

no correlation was found between trochanter femur BMD and ba-PWV, nor between BMI and ba-PWV (Table 2). Age was the factor most strongly correlated to ba-PWV $(r=0.586, \mathrm{P}<0.001)$.

\section{Multivariable Analysis}

In addition to statistically significant outcome variables from the univariate analysis, potential clinically confounding variables (alcohol intake, smoking status, and exercise) were used as covariables in the regression analysis. Independent of these factors, ba-PWV was shown to be significantly and negatively correlated with L-spine BMD but was not significantly associated with BMD in the femoral neck or total femur (Table 3). Age was the factor most strongly predictive of ba-PWV; a relationship was also observed between metabolic syndrome and baPWV on multivariate analysis (Table 3).

\section{DISCUSSION}

This study evaluated the correlation between arterial stiffness and BMD in healthy women undergoing regular medical checkup. Multivariate analysis identified an independent, significant, and negative correlation between ba-PWV, a measure of vascular stiffness, and lumbar spine BMD. Metabolic syndrome was also significantly correlated with ba-PWV, while age was the variable most strongly correlated with PWV.

The correlation between BMD and vascular stiffness has not hitherto been clearly established by epidemiological or clinical studies including the study by the Japanese researchers. Age, smoking, hypertension, diabetes, hyperlipidemia, renal failure, 
physical activity, and menopause are risk factors for both conditions. In addition, inflammatory factors, such as oxidized low density lipoprotein, osteopontin, and osteoprotegerin, as well as other substances that affect bone health, such as vitamin D and estrogen, are expected to influence the relationship between BMD and vascular stiffness. ${ }^{17-20)}$

A correlation between osteoporosis and increased ba-PWV has been observed during a study to determine the association between lumbar BMD and ba-PWV in postmenopausal women. ${ }^{9)}$ As in this study, a significant negative correlation was also found between lumbar BMD and vascular stiffness-measured by both AI and PWV-among obese African-American women $(\mathrm{P}=0.01)$; though the relationship was only marginally significant, vascular stiffness was also inversely proportional to the femoral neck BMD $(\mathrm{P}=0.05)$ and total hip $\mathrm{BMD}(\mathrm{P}=0.06) .{ }^{10}$

Frost et al. ${ }^{21)}$ found that PWV was significantly inversely proportional to femoral BMD but was not significantly correlated with lumbar BMD among postmenopausal women. This result is probably due to osteophyte, osteosclerosis, osteophytosis, or vascular calcification. Women may experience a decrease in spongy bone postmenopausally; since spongy bone distribution varies between the L-spine and the femoral neck, a reduction in BMD may first be noticed in the spine and only later in cortical and spongy bone, such that lower femoral BMD is not evident until the age of 70 years. One study of a Japanese population also found that lumbar BMD began to decrease in women in their 50s, around the time of menopause. ${ }^{22)}$ The population in this study comprised a group of women aged 53 years, on average, who were experiencing postmenopausal bone loss; therefore, a significant correlation was observed between lumbar BMD and PWV. The fact that degenerative arthritis and vascular calcification are more likely to occur with advancing age may explain the weak correlation with lumbar BMD and stronger correlation with femoral BMD in older patient cohorts.

In this study, a significant correlation was observed between features of metabolic syndrome and ba-PWV. Consistent with this finding, multiple Asian studies have reported that metabolic syndrome leads to significantly higher PWV, especially among women. ${ }^{23,24)}$ The results of our study should be interpreted with caution because BMI, instead of waist circumference, was used to classify metabolic syndrome. However, PWV, which is a measure for arterial stiffness and atherosclerosis, may increase due to $\mathrm{BP}$, diabetes, and hyperlipidemia and is probably correlated with metabolic syndrome, as found in previous research. Further research should be conducted because ba-PWV has rarely been researched in healthy adults without hypertension, diabetes, or hyperlipidemia, because BP can act as a confounding factor, and because no definite conclusion has been drawn about correlation with metabolic syndrome in which insulin resistance and body fat are regarded as important factors.
With age comes the development of arterial stiffness and an often remarkable rise in systolic BP. Here, the finding that baPWV was proportional to age is consistent with the fact that arterial stiffness is proportional to age.

We acknowledge some limitations to this study. Subject data was obtained from chart review and was limited with respect to menopausal status. As the decrease in estrogen production after menopause can contribute to the development of atherosclerosis and osteoporosis, menopausal status could have been a confounding factor in our analysis. In addition, osteoporosis can be caused by calcium and vitamin D deficiency and some researchers have reported that calcium and vitamin $\mathrm{D}$ levels are correlated with arterial stiffness; ${ }^{25)}$ however, no consideration was given to these nutrients in this study.

In conclusion, our analysis demonstrated that lumbar spine BMD was independently and significantly correlated with baPWV in women after correction for age, metabolic syndrome, alcohol intake, and exercise status $(\mathrm{r}=-0.229, \mathrm{P}=0.01)$. Further epidemiological and clinical research should be conducted to investigate the correlation between BMD and arterial stiffness in men and women.

\section{CONFLICT OF INTEREST}

No potential conflict of interest relevant to this article was reported.

\section{REFERENCES}

1. Von der Recke P, Hansen MA, Hassager C. The association between low bone mass at the menopause and cardiovascular mortality. Am J Med 1999;106:273-8.

2. Kado DM, Browner WS, Blackwell T, Gore R, Cummings SR. Rate of bone loss is associated with mortality in older women: a prospective study. J Bone Miner Res 2000;15:1974-80.

3. Marcovitz PA, Tran HH, Franklin BA, O'Neill WW, Yerkey M, Boura J, et al. Usefulness of bone mineral density to predict significant coronary artery disease. Am J Cardiol 2005;96:1059-63.

4. Magnus JH, Broussard DL. Relationship between bone mineral density and myocardial infarction in US adults. Osteoporos Int 2005;16:205362.

5. Hak AE, Pols HA, van Hemert AM, Hofman A, Witteman JC. Progression of aortic calcification is associated with metacarpal bone loss during menopause: a population-based longitudinal study. Arterioscler Thromb Vasc Biol 2000;20:1926-31.

6. Schulz E, Arfai K, Liu X, Sayre J, Gilsanz V. Aortic calcification and the risk of osteoporosis and fractures. J Clin Endocrinol Metab 2004;89: 4246-53.

7. Tanko LB, Bagger YZ, Christiansen C. Low bone mineral density in the hip as a marker of advanced atherosclerosis in elderly women. Calcif Tissue Int 2003;73:15-20.

8. Hirose K, Tomiyama H, Okazaki R, Arai T, Koji Y, Zaydun G, et al. Increased pulse wave velocity associated with reduced calcaneal quanti- 
tative osteo-sono index: possible relationship between atherosclerosis and osteopenia. J Clin Endocrinol Metab 2003;88:2573-8.

9. Mikumo M, Okano H, Yoshikata R, Ishitani K, Ohta H. Association between lumbar bone mineral density and vascular stiffness as assessed by pulse wave velocity in postmenopausal women. J Bone Miner Metab 2009;27:89-94.

10. McFarlane SI, Qureshi G, Singh G, Venner-Jones K, Salciccioli L, Lazar J. Bone mineral density as a predictor of atherosclerosis and arterial wall stiffness in obese African-American women. Cardiorenal Med 2012;2:328-34.

11. Yamashina A, Tomiyama H, Takeda K, Tsuda H, Arai T, Hirose K, et al. Validity, reproducibility, and clinical significance of noninvasive brachial-ankle pulse wave velocity measurement. Hypertens Res 2002;25: 359-64.

12. Sugawara J, Hayashi K, Yokoi T, Cortez-Cooper MY, DeVan AE, Anton MA, et al. Brachial-ankle pulse wave velocity: an index of central arterial stiffness? J Hum Hypertens 2005; 19:401-6.

13. Tomiyama H, Koji Y, Yambe M, Shiina K, Motobe K, Yamada J, et al. Brachial-ankle pulse wave velocity is a simple and independent predictor of prognosis in patients with acute coronary syndrome. Circ J 2005;69:815-22.

14. Kitahara T, Ono K, Tsuchida A, Kawai H, Shinohara M, Ishii Y, et al. Impact of brachial-ankle pulse wave velocity and ankle-brachial blood pressure index on mortality in hemodialysis patients. Am J Kidney Dis 2005;46:688-96.

15. Meguro T, Nagatomo Y, Nagae A, Seki C, Kondou N, Shibata M, et al. Elevated arterial stiffness evaluated by brachial-ankle pulse wave velocity is deleterious for the prognosis of patients with heart failure. Circ J 2009; 73:673-80.

16. Lee HT, Shin J, Lim YH, Kim BK, Kim YT, Lee JU, et al. The relationship between coronary artery calcification and bone mineral density in pa- tients according to their metabolic syndrome status. Korean Circ J 2011; 41:76-82.

17. Gourdy P, Calippe B, Laurell H, Tremollieres F, Douin-Echinard V, Lenfant $\mathrm{F}$, et al. Role of inflammatory cytokines in the effect of estradiol on atheroma. Clin Exp Pharmacol Physiol 2008;35:396-401.

18. Maziere C, Louvet L, Gomila C, Kamel S, Massy Z, Maziere JC. Oxidized low density lipoprotein decreases Rankl-induced differentiation of osteoclasts by inhibition of Rankl signaling. J Cell Physiol 2009;221:572-8.

19. Demer LL. Vascular calcification and osteoporosis: inflammatory responses to oxidized lipids. Int J Epidemiol 2002;31:737-41.

20. Browner WS, Lui LY, Cummings SR. Associations of serum osteoprotegerin levels with diabetes, stroke, bone density, fractures, and mortality in elderly women. J Clin Endocrinol Metab 2001;86:631-7.

21. Frost ML, Grella R, Millasseau SC, Jiang BY, Hampson G, Fogelman I, et al. Relationship of calcification of atherosclerotic plaque and arterial stiffness to bone mineral density and osteoprotegerin in postmenopausal women referred for osteoporosis screening. Calcif Tissue Int 2008;83:112-20.

22. Yoshimura N, Kinoshita H, Danjoh S, Takijiri T, Morioka S, Kasamatsu $\mathrm{T}$, et al. Bone loss at the lumbar spine and the proximal femur in a rural Japanese community, 1990-2000: the Miyama study. Osteoporos Int 2002;13:803-8.

23. Weng C, Yuan H, Tang X, Huang Z, Yang K, Chen W, et al. Age- and gender dependent association between components of metabolic syndrome and subclinical arterial stiffness in a Chinese population. Int J Med Sci 2012;9:730-7.

24. Kim YK. Impact of the metabolic syndrome and its components on pulse wave velocity. Korean J Intern Med 2006;21:109-15.

25. Giallauria F, Milaneschi Y, Tanaka T, Maggio M, Canepa M, Elango P, et al. Arterial stiffness and vitamin D levels: the Baltimore longitudinal study of aging. J Clin Endocrinol Metab 2012;97:3717-23. 